\title{
Ciudadanía en los jóvenes de las universidades de Lima. Una propuesta de fortalecimiento de la ciudadanía a través de la enseñanza de la historia social y política'
}

Recibido: 03/10/2016

Aprobado: 07/11/2016
Carlota Casalino

Universidad Nacional Mayor de San Marcos

< ccasalinos@unmsm.edu.pe >

David Atarama Joel Castro ${ }^{\star}$

no les formamos para responder a las preguntas de ayer, sino a aquellas que tendrán que responder mañana, les formamos para que estas respuestas respeten un conjunto de principios y de valores alrededor de la ciudadanía democrática y de los derechos del hombre.

(Audigier 1999: 6)

\section{RESUMEN}

Partimos del problema que en el país hay poca conciencia ciudadana, especialmente entre los jóvenes, quienes se desinteresan por los asuntos públicos. Proponemos que como la ciudadanía es resultado de un proceso histórico, a través de la educación —motor de progreso- podemos construirla y de esa forma fortalecer una ciudadanía activa. Además señalamos que la Historia puede contribuir a formar conciencia ciudadana través de la enseñanza de temas de historia social y política que evidencia problemas actuales que han persistido a lo largo del tiempo, contribuyendo de esta manera — además - a cerrar brechas entre la historia académica y la enseñanza de la historia. Luego, definimos de manera básica ciudadanía para luego mostrar evidencia empírica acerca de las percepciones que tienen algunos jóvenes de tres universidades de Lima sobre este tema.

Palabras clave: ciudadanía, jóvenes universitarios, educación, enseñanza de la Historia.

\section{Citizenship among young people of universities of Lima. A proposal of teaching social and political history to build civic consciousness}

\begin{abstract}
We start from the problem that in the country there is little level of civic consciousness, especially among young people, who lose interest in public affairs. We propose that as citizens are the result of a historical process, through education — which is the engine of progress - we can build and thus strengthen active citizenship, especially among young people. We also note that de course of History can help build civic consciousness by teaching social and political processes that show current problems that have persisted over time. This will be the manner of contributing also to close the gap between academic history and the teaching of history. Then, we define in the basic way what is citizenship and then show empirical evidence about the perceptions of some young people from three universities in Lima on this subject.
\end{abstract}

KEYwORDs: Citizenship, university students, education, teaching history.

1 Este artículo es parte de la investigación desarrollada en el IIHS en el ańo 2014 con registro N. 141501225.

2 Participaron como ayudantes de investigación los siguientes estudiantes: Juan Marcos Martínez, Joel Segura, Ángela Quispe, Madeleine Torres, Juan Diego Villegas, Cristhian Gómez, Lorenzo Huamaní, Renzo Loza, Alberto Navarrete y Ana Laya. 


\section{Importancia de la educación y de la enseñanza de la Historia para el fortalecimiento de la ciudadanía en la actualidad}

D aron Acemoglu y James Robinson (2012) señalan que varios países han fracasado porque tienen instituciones políticas y económicas extractivistas, mientras que los países que se han desarrollado tienen instituciones políticas y económicas inclusivas. Explican, además, que aquellos países que lograron desarrollar instituciones inclusivas lo hicieron porque vivieron procesos históricos en los cuales tuvieron que resistirse a los intereses particulares, oligárquicos y colonizadores, donde una minoría se beneficiaba a costa de la mayoría. Incluso tuvieron que aprovechar las crisis políticas y económicas como oportunidades para optar por instituciones inclusivas, además de evitar que se impusiera la ley de hierro de la oligarquía. De esa manera, con instituciones inclusivas generaron procesos de destrucción creativa y desarrollaron ciclos virtuosos donde todos y todas construyeron las condiciones para desarrollarse (Acemoglu \& Robinson 2012: 95-105).

Desde sus orígenes nuestro país ha tenido instituciones extractivistas, y cada crisis política o económica no fue aprovechada para reemplazar estas instituciones por otras que sean inclusivas, sino que restablecieron y reprodujeron instituciones extractivistas, haciendo que nuestra historia se transforme en un círculo vicioso de desigualdad, inequidad y ausencia de oportunidades (Acemoglu \& Robinson 2012: 504-506). ¿Qué hacer para cambiar ese círculo vicioso? El aprendizaje que se desprende de la propuesta de Acemoglu y Robinson es considerar que una clave para construir instituciones inclusivas hoy en día radica en el desarrollo de la ciudadanía. Una ciudadanía que en el marco de un régimen democrático asuma de manera responsable su condición de soberana. Una ciudadanía republicana que aspire a construir una comunidad política en la cual todos sus integrantes tengan las mismas oportunidades para desarrollarse, sobre la base de la construcción de instituciones políticas inclusivas y estables. ¿Cómo lograr en nuestro país esa ciudadanía comprometida con su comunidad política? Un paso para lograr ello es a través de la educación, considerado como uno de los motores de prosperidad por los autores citados. Demostrarlo es el objeto de este trabajo.

Para ello partimos de vincular la democracia con la educación sosteniéndonos por lo tanto en la tradición republicana. Esta tradición considera que la educación es la estrategia más adecuada para formar valores, cultivar la virtud cívica y formar ciudadanos responsables y conscientes de su rol histórico en la construcción de la comunidad política. En esta perspectiva Javier Peńa (2000) sostiene que la buena ciudadanía, o los ciudadanos virtuosos no surgen de manera espontánea en una sociedad, sino que deben coincidir instituciones y virtud ciudadana a través de la educación cívica.

Así, es la propia sociedad que necesita que sus ciudadanos se involucren en los asuntos públicos con responsabilidad y a favor del bien común. De igual parecer es Ramón Ruiz Ruiz (2009) al sostener el concepto de "universalismo" consistente en una deliberación entre iguales - para alcanzar acuerdos sustantivos- y la participación intensa de los ciudadanos. Para ello se puede apelar a la virtud cívica, la misma que puede acrecentarse bajo ciertas circunstancias, siendo el principal instrumento la educación. Asimismo, el papel central de la virtud cívica como articulador de la sociedad es analizado por Luz Margarita Cardona (2011) quien explica el renovado interés por la tradición republicana dadas las limitaciones que presenta hoy en día el diseño democrático liberal ahondada por la despolitización y privatización de la vida pública promovida por el neoliberalismo.

La educación es un valor fundamental de toda sociedad moderna, así lo expresa Fernando Savater (2004) en su obra "El valor de Educar". Este autor considera que es muy importante discutir y promover el fortalecimiento de la educación y valorarla positivamente como la clave para construir una mejor sociedad. Así, considera que el problema educativo es remediable y por lo tanto hay que poner manos a la obra. Sobre la base de esa actitud optimista frente a los problemas de la educación, Savater considera que hay esperanza en tanto consideremos que los seres humanos tenemos una capacidad innata para aprender unos de otros y por lo tanto podemos mejorarnos. Desde esa perspectiva, pero no desde el campo de la filosofía, sino desde el campo de la psicología, Rosario Ortega (2005) sostiene que gracias a la educación, los seres humanos entramos en contacto con los conocimientos que han acumulado nuestros predecesores sin necesidad de comenzar nuestro aprendizaje desde cero. En el caso del aprendizaje de los adultos, se establece que este grupo sigue en permanente desarrollo y formación.

Del Valle Ballón (2012) afirma que el acceso a la información y al conocimiento es incluso más preciso y 
más seguro, ya que el adulto al ser independiente, busca conscientemente aquella información y conocimiento que le interesa. De esa manera este nuevo conocimiento es muy valioso. Otra ventaja es que el adulto se compromete personalmente con su propio desarrollo, y la regularidad en el cumplimiento de las tareas, se autodetermina, posee pensamiento abstracto y tiene más experiencia. Para Del Valle Ballón el adulto temprano se ubica entre los 18 a los 24 ańos, el adulto medio a partir de los 40 años y el adulto mayor a partir de los 60 años.

La educación de los ciudadanos es el tema de reflexión de Manuel Iguiñiz y Arturo Miranda (2011), quienes analizan la política educativa en Lima metropolitana, identifican las principales dificultades, contradicciones y retos que significa la descentralización de la educación y la situación de la educación en Lima metropolitana. Es a partir de ese diagnóstico que se puede identificar por dónde proponer el fortalecimiento de una ciudadanía virtuosa sobre la base de temas históricos y políticos clave.

A su vez, Augusta Valle reflexiona sobre el conocimiento histórico y la escuela (Valle 2013), indicando los principales problemas que hay cuando se enfoca el estudio de la historia en el ámbito escolar desde una perspectiva tradicional, es decir recordar fechas y nombres, máxime si la propuesta educativa neoliberal vigente en nuestro país desde la década de 1990 confunde la formación de seres humanos con la formación de recursos. Valle sostiene que es necesario disminuir la distancia entre historia académica e historia escolar, modificar el Diseño Curricular Nacional (DCN) para que aborde la historia como una materia en la cual se desarrollen diversas capacidades, donde la más importante es poder reconocerse como ser histórico, con un pasado, con un presente y con proyección de futuro. Son tres las capacidades que sostiene se deben desarrollar: el pensamiento histórico, consciencia histórica e identificarse como seres históricos.

En esa perspectiva, Lima, Bonilla y Arista (2010) analizan la enseńanza de la historia en la escuela mexicana, identifican que hasta 1993 la historia era una materia sustentada en el aprendizaje memorístico, luego a partir de ese ańo y con la reforma de los planes y programas se inició la aplicación de un enfoque formativo en la enseńanza de la historia. Ello significa que con la historia se enseña a pensar históricamente, se prioriza la explicación, se facilita la comprensión multicausal del presente, se promueve el análisis crítico de la información y se fortalece la identidad nacional.
Es en este punto que cobra importancia la historia, porque el conocimiento de nuestra historia nos permite saber lo que nuestros antepasados han realizado, cómo lo hicieron y por qué. De ahí que la enseñanza de la historia social y política es clave cuando queremos fortalecer a las y los ciudadanos para que se reflexione y se tome conciencia de nuestra responsabilidad como constructores de la comunidad política.

Respecto a la experiencia de la enseñanza de la historia tenemos el libro de Josep Fontana (1999) titulado "Enseñar historia con una guerra civil por medio" quien compara un texto de enseñanza de la historia elaborado durante la primera república espańola (en 1933) y un texto de enseńanza de la historia utilizado durante el período franquista (1936). De la comparación de ambos textos uno puede apreciar cuál es el sentido, propósito y contenidos de la enseńanza de la historia para construir la comunidad política que aspiramos. Así, uno puede valorar la importancia de la enseñanza de la historia para formar ciudadanos con sentido histórico y comprometido con su comunidad, o la enseńanza de la historia para ratificar el sistema inequitativo y autoritario anulando la posibilidad de formar ciudadanía.

En esa misma línea, Marc Ferro (1998) analiza cómo se cuenta la historia a los niños en el mundo entero. Explica que en un mundo cada vez más globalizado la historia de cada sociedad ha cobrado mayor relevancia. Así, hay interés en controlar el pasado y legitimar dominaciones (como en Sudáfrica). Un papel central en revertir ello es el proceso de descolonización, que significó la revisión de la historia patria desde una nueva perspectiva. Ferro señala que la historia ejerce una doble función, terapéutica y militante.

Nicolás Cueva (1991) considera que es importante estudiar la historia porque esta adquiere una función ideológica. Así, los grupos de poder utilizaron la historia para negar la conciencia colectiva de los sectores populares. Sin embargo, hoy en día, según Cueva, la enseńanza de la historia debe estar orientada a desarrollar conciencia crítica y a afianzar nuestra identidad nacional. En ese sentido, y siguiendo a Alberto Flores Galindo, este autor sostiene que el profesor tiene la posibilidad de contribuir en el proceso de formación, consolidación y desarrollo de la identidad histórica de los estudiantes. Un tema particularmente relevante, es la enseñanza de la historia cuando el país ha vivido procesos de violencia estructural. 
Al respecto Susana Finocchietti (1999) considera que los textos escolares contienen elementos no adecuados para abordar este problema de la violencia, por lo que no hay una verdadera comunicación de los procesos históricos. La autora critica que prevalezca una historia de los acontecimientos, repetitivo plagado de nombres propios, fechas y una sucesión de lugares sin la explicación ni el contexto que permita comprender los procesos históricos de manera crítica y reflexiva. Propone que se promuevan experiencias en las cuales los estudiantes tengan acceso a fuentes primarias, visitar lugares históricos o museos y propiciar lecturas que promuevan experiencias afectivas donde los estudiantes se sientan involucrados como actores de la historia.

Asimismo, Carlos Rojas (1989) enfatiza la importancia de la motivación al momento de la enseñanza de la historia. Sin motivación no se despierta el interés del estudiante, ello impedirá la participación consciente en la clase. Es fundamental enseñar en un clima donde el estudiante esté motivado y estimulado para participar durante la sesión.

Respecto a los contenidos o temas de historia que se enseñan, María Paula González (2011) introduce el concepto transposición didáctica utilizado por Chevallard, que consiste en que todo proyecto social de enseñanza se construye con la designación de un conjunto de saberes como contenidos a enseńar. A partir de ello, los saberes a enseñar sufren un conjunto de transformaciones adaptativas que van a hacerlos aptos para ocupar un lugar entre los objetos de enseñanza. El trabajo que transforma un "objeto de saber a enseńar" en un "objeto de enseñanza" es denominado transposición didáctica. Sin embargo, ella sostiene que esa transposición didáctica solo legitima la distancia entre la historia académica y la escolar, e incluso la traiciona, por ello considera que no debe haber distancia entre una y otra, sino que se debe hacer el esfuerzo para que esa brecha no se produzca.

La tesis de Patricia Escobar (1991) sostiene que el estudiante debe involucrarse descubriendo la realidad donde está inmerso. Por ello si el estudiante "aprende haciendo" logra formarse de manera integral, pues aprende a ser crítico, reflexivo y analítico. Debe priorizar una sucesión cronológica, selectiva analítica, y desarrollar capacidad crítica y creativa frente a la realidad. Las variables indispensables a enseñarse en el curso de Historia son el quién, el dónde, el cómo y el por qué, con esas variables el estudiante está habilitado para reconstruir la historia. Los temas deben incluir las luchas, retrocesos, fracasos y progresos de los hombres en sociedad, ello considera a las minorías dirigentes y también a las gentes anónimas. Todo lo cual contribuye a comprender el mundo que se vive y a construir el futuro. Para comprender y hacer comprender la historia, el estudiante debe tener la experiencia de observar, reflexionar, imaginar y crear. Además es preciso promover en el aula el debate, la discusión y el diálogo con el fin de contribuir a que el estudiante madure su espíritu crítico. Escobar sostiene que el objetivo de la enseńanza de la historia es la formación de la conciencia histórica. Ello significa que el alumno desarrolle un sentido de pertenencia.

Si pasamos revista a la bibliografía que desarrolla temas de historia social y política y que fue producida en el ańo 2013, podemos apreciar que contamos con un conjunto de temas y problemas que es necesario que trasciendan el ámbito meramente académico, ya que pueden ser una buena oportunidad para reflexionar sobre los problemas, obstáculos -algunos estructurales y otros coyunturales- que impiden que nuestro país se desarrolle. Por ejemplo, hoy en día se puede conocer el papel que ha jugado la corrupción para impedir el desarrollo de nuestro país, gracias al trabajo de Alfonso Quiroz (2013). Así, sostiene que los "sistemas menos desarrollados enfrentan los dilemas interrelacionados de cómo permitir y promover el crecimiento, diseñar y hacer cumplir Constituciones que favorezcan la estabilidad y el desarrollo, distribuir el ingreso de modo más equitativo, democratizar y equilibrar el poder político, establecer el imperio de la ley y educar a los ciudadanos dentro de una sociedad civil vigorosa, que supervise una eficiente administración estatal. Los agentes corruptos minan estos esfuerzos, a veces con consecuencias y costos devastadores".

Tomar conciencia del peso de la corrupción, no como un mero accidente de la década de 1990, sino como un problema estructural, puede ayudarnos a reflexionar en la necesidad de contraponer la tolerancia hacia la corrupción existente en nuestra cultura política, promoviendo el cultivo de la virtud cívica. Además nos da argumentos históricos para persuadir a las nuevas generaciones que es indispensable tomar conciencia que cada uno de los integrantes de esta sociedad somos agentes históricos, por lo que de nosotros depende tomar la iniciativa para romper con la reproducción de la corrupción grande y pequeńa en nuestro país.

Otro tema, que nos ayuda a reflexionar sobre las características de nuestra ciudadanía es el trabajo de Alicia del Águila (2013) quien analiza la ciudadanía corporativa que se constituyó en el siglo XIX en nues- 
tro país. Reflexionar al respecto, el peso y papel de los cuerpos sociales decimonónicos, como la Iglesia, los militares, los gremios, las comunidades de indígenas, nos ayudará a comprender mejor nuestros procesos históricos y los retos que enfrentamos como comunidad. Este trabajo es singularmente importante, porque sienta las bases para reflexionar sobre la ciudadanía desde una perspectiva histórica. Es decir, la ciudadanía se construye, tiene un punto de partida histórico, y por lo tanto, hoy en día debemos seguir construyéndola, sobre la base de lo que la historia nos enseña.

Respecto al siglo XX hay importantes estudios que nos permiten tener una visión más compleja y completa de nuestra historia social y política. Así, el libro de Pease y Romero (2013) puede ayudar a comprender mejor los hitos políticos cruciales de nuestra historia. Las características particulares del Estado peruano, los poderes políticos locales, las instituciones políticas, la exclusión persistente de la mayoría de la población durante buena parte del siglo XX. Con el libro de Valladares Quijano (2013) podemos apreciar coyunturas políticas clave, que abren paso a períodos de transición política producto de la presión del movimiento popular. A partir del trabajo de Mario Meza (2013) se puede conocer el ejercicio de la justicia y el poder en tiempo de violencia a partir de tres categorías como el orden, la seguridad y la autoridad. Estos son algunos de los temas de Historia Social e Historia Política que pueden ser relevantes para ser desarrollados en el ámbito de la educación formal y en la no formal, con el ánimo de promover la reflexión y la toma de conciencia de nuestro rol como ciudadanos producto de nuestro pasado, actores del presente y responsables del futuro de nuestra comunidad política. Fernández-Maldonado (2015) señala la importancia de los jóvenes como agentes de cambio, pero desde una mirada crítica, desde su perspectiva generacional. Con este trabajo podemos acercarnos al conocimiento sobre los temas por los cuales los jóvenes hoy en día se movilizan. Es necesario transformar en historia ese movimiento juvenil que luego de cinco marchas masivas, organizadas e innovadoras, hicieron retroceder - como nunca antes - una norma ya aprobada y promulgada. Al ganar la opinión pública y al demostrar que estaban decididos, fueron un punto de quiebre y de inicio de una coyuntura crítica, que puede ser el inicio para la construcción de esas instituciones inclusivas referidas al inicio de este trabajo.

En síntesis, en la historia social y política hay suficiente conocimiento académico reciente que puede ser difundido en la educación formal y no formal que permita reflexionar y tomar conciencia de la importancia de fortalecer la virtud cívica a través de la educación. Temas como la presencia y crisis de la mediación política, la persistencia del caudillismo, el problema de la corrupción, los procesos de transición política, los hitos de crisis y refundación de la república, o las características de la ciudadanía, de la sociedad civil o de los jóvenes, pueden ser abordados en un proyecto educativo. De esa manera la historia académica cobrará mayor sentido y protagonismo en el papel formador de la ciudadanía en nuestro país.

En esa perspectiva, a continuación vamos a desarrollar algunos conceptos sobre la ciudadanía, notando la complejidad del término y la manera como su significado obedecerá a diversas tradiciones, donde destacan la liberal y la republicana. Luego, expondremos las percepciones que hoy en día tienen los jóvenes universitarios acerca de la ciudadanía y la participación en los asuntos públicos.

\section{La ciudadanía $\mathbf{a}^{*}$}

La ciudadanía es la cualidad de ciudadano. Al respecto, la academia sostiene que la ciudadanía comprende la totalidad de los miembros de un ente político común y su estatus como miembros de pleno derecho. Además, consideran que es fundamental la pertenencia al Estado-nación a través de la nacionalidad. A partir de esa condición, la ciudadanía otorga a quien lo detenta un estatus seguro con los mismos derechos fundamentales civiles, políticos y sociales (Rieger 203). Es decir, aquel que tiene ciudadanía posee garantías para tener igualdad ante la ley, libertad de conciencia, libertad de asociación, sufragio, entre otros. El acceso a ello es delimitado por la norma constitucional (Casalino 2010: 8).

Se trata de un concepto que tiene diversos enfoques, entre los que destacan la concepción liberal y la republicana. En el caso de la primera, Rieger sostiene que el liberalismo considera al ciudadano definido esencialmente por los derechos de su libertad individual como derechos de defensa frente al Estado, esta perspectiva delimita y controla el poder, además, los derechos sociales tienen el objetivo de asegurar la autonomía individual. Desde esta perspectiva, el ciudadano

3 Este acápite está basado en un documento que elaboré hace un tiempo y que sirvió para desarrollar cursos sobre ciudadanía dirigidos a diversos sectores sociales y políticos. 
es protegido hasta del mismo Estado (Rieger 203-204). Mientras que desde la segunda, el ciudadano se realiza a través de su compromiso a favor del bien común y la participación en la dominación, es decir aspira a cultivar la virtud cívica (Casalino 2010: 8).

Estas perspectivas en muchos aspectos no son complementarias, sino que se relacionan en tensión. Estas tensiones se pueden apreciar especialmente cuando se busca diseñar instituciones en las cuales prevalezca la libertad, la igualdad y la solidaridad; asimismo, entre la orientación hacia el bien común y los derechos individuales; como también entre los derechos humanos y los derechos civiles; y entre los ciudadanos como comunidad política y las pertenencias étnicas, religiosas, entre otras (Rieger 204).

La ciudadanía moderna es un producto histórico, Sinesio López sostiene que al menos hay cuatro características básicas que definen al ciudadano, las mismas que se han ido desarrollando en el tiempo. Estas son la existencia de individuos, el tener derechos, que el Estado ofrezca garantías a los derechos; y finalmente, la existencia de una comunidad política (López 1977:118). En ese sentido, define al ciudadano como:

... un individuo o comunidad de individuos con derechos garantizados por el Estado y con responsabilidades hacia la comunidad política de la que forma parte (Sinesio López 1997: 118-119).

Este mismo autor sostiene que la emergencia de la ciudadanía supone un cambio en las relaciones de autoridad, en la que los gobernados reivindican y desarrollan un conjunto de derechos y responsabilidades frente a los gobernantes, que terminan aceptándolos. En ese sentido, se invierte la manera como hasta ese momento se había organizado el poder, sustentado en privilegios y facultades ejercidas por los gobernantes en desmedro de los gobernados (Casalino 2010: 10).

Para que esas transformaciones se realicen, tuvo que producirse profundos cambios culturales, jurídicos, éticos, políticos, económicos y sociales. Entre los procesos más importantes podemos mencionar el de secularización de la sociedad, el proceso de individualización y el surgimiento de la democracia moderna (López 1997: 28). Asimismo, se requieren determinadas condiciones para la existencia de la ciudadanía moderna como por ejemplo cultura cívica, secularización, nuevo esquema administrativo del Estado, declinación de valores particularistas, emergencia de la idea del es- pacio público, existencia de prensa política escrita y opinión pública, conceptos que nos remiten a la modernidad política (Casalino 2010: 10).

La ciudadanía moderna surge a partir del siglo XVIII, cuando en Europa se inició la conquista de los derechos civiles, por ejemplo en torno a la idea de libertad. Luego en el siglo XIX se consiguieron derechos políticos como la participación política ampliada por el sufragio universal. En el siglo XX se conquistaron los derechos sociales, que comprenden la participación en el bienestar producido por la sociedad (López 1997: 29). En la actualidad la relación entre ciudadanía y Estado es central, en el sentido que se trata que por un lado, el Estado garantice los derechos y por el otro lado, que los ciudadanos los ejerzan y los defiendan.

A mediados del siglo XX, T. H. Marshall sostuvo que el desarrollo de la ciudadanía democrática incluye tres elementos: la ciudadanía civil, la política y la social. Para este autor, la condición de ciudadano, titular de la plenitud de derechos públicos subjetivos civiles, políticos, económicos, sociales y culturales, son otorgados por el sistema jurídico.

En el caso de la ciudadanía civil, en el siglo XVIII se dio origen a los derechos vinculados a la libertad personal y la autonomía de las personas, como por ejemplo los derechos de propiedad, de compra-venta, la libertad de movimiento, de expresión, de organización, de palabra, de pensamiento y de fe religiosa; así como el derecho a la justicia. Esto significó el quiebre de una sociedad organizada en cuerpos sociales, para dar lugar a una sociedad basada en individuos, de los cuales, un grupo de ellos accedía a estos derechos.

La ciudadanía política se produce en el siglo XIX y dio lugar al derecho a elegir y ser elegido en el manejo del poder político a través del sufragio universal. Marshall señaló: "Por el elemento político entiendo el derecho a participar en el ejercicio del poder político, como miembro investido con autoridad política, o como elector de dicho cuerpo" (Marshall 1965: 23).

La ciudadanía social corresponde al siglo XX, dio origen a los derechos económicos y sociales gracias a los cuales los ciudadanos tienen derecho a compartir todo el patrimonio social y a vivir la vida de un ser civilizado según los patrones que prevalezcan en la sociedad. Marshall supuso que la ciudadanía social permitiría eliminar las desigualdades y borraría algunas diferencias de estatus, lo que implica el desarrollo de la igualdad social y de la solidaridad (López 1997: 91 y Gutiérrez 2000: 170). Según Heater, Marshall sostuvo que los derechos 
sociales tienen una naturaleza distinta de los civiles o políticos, pues mientras que estos últimos pueden definirse y reconocerse con cierta precisión (por ejemplo el derecho a un juicio o el derecho al voto), los derechos sociales atañen a la calidad de vida. El acceso a la educación o al sistema de salud, son derechos sociales, pero el principio de ciudadanía social no puede prescribir el nivel que cabe esperar de escuelas y hospitales. Así, detentar estos derechos sociales es básico para el disfrute efectivo de los derechos civiles y políticos, pues la pobreza y la ignorancia merman inevitablemente el deseo y la oportunidad de poder beneficiarse de ellos.

Como se puede apreciar, los elementos de ciudadanía civil, política y social corresponden al proceso histórico del mundo europeo occidental, de manera particular a aquella que está vinculada al ciudadano hombre, trabajador y blanco. En estos elementos de ciudadanía no está incluida la historia social correspondiente a la mujer, ya que esta no alcanzó en esa misma época la ciudadanía ni civil ni política. Tampoco está el caso peruano, dado que en nuestro país estos derechos se produjeron casi en simultáneo entre el siglo XIX y XX (Casalino 2010: 10).

En nuestro caso, ingresamos a la modernidad política mediatizados por la presencia del Virrey Abascal a partir de la crisis de 1808. En ese entonces, se iniciaron los primeros derechos civiles y políticos especialmente el derecho a la libertad de prensa y el derecho al sufragio, pero fueron intermitentes y dependientes de cómo se desarrolló el proceso político en la Metrópolis (Peralta 2010-capítulo 4). En el período republicano, los primeros derechos civiles fueron reconocidos durante las tres primeras décadas del siglo XX, luego a partir de la década del cincuenta se demandaron los derechos sociales, junto a ellos se demandaron de manera simultánea derechos civiles y políticos. Ello constituye una primera diferencia respecto a la experiencia europea, pero hay otras dos: el hecho que las demandas sociales en el caso peruano provienen de la pobreza de la población y que no se logró conformar una comunidad política nacional en la que estén plenamente integrados los sectores populares. Todo lo cual configuró el carácter inacabado del proceso de formación de la ciudadanía en nuestro país. Por esa razón, Sinesio López sostiene que las deficiencias en la formación de la ciudadanía se deben a la acentuada desigualdad de esta que hace que unos peruanos sean más ciudadanos que otros en el acceso desigual al conjunto de los derechos civiles, políticos y sociales, dando lugar a una alta inconsistencia ciudadana y la formación de brechas ciudadanas, sociales, regionales, rural-urbanas, de género y étnicas (López 1997: 29).

Adicional a ello, este mismo autor sostiene la existencia de dos matrices o tradiciones en la conformación de la ciudadanía en el Perú. Por un lado está el estatismo comunitarista con un sentido progresista; y por el otro el liberalismo que tenía un sentido conservador sustentado por la oligarquía peruana (López 1997: 33). Esto es central, ya que tanto la tradición comunitarista como la liberal están presentes en el proceso de configuración de la ciudadanía en el Perú.

La ciudadanía en el Perú, al tender hacia la universalización y a homogeneizar las diferencias produjo -paradójicamente- ciudadanos diferenciados. La universalización señalada se produjo a partir de los siguientes principios: dignidad igual para todos, pertenencia a la misma comunidad política, trato igual para todos a través de las mismas leyes. En ese sentido, siguiendo a Sinesio López, algunos ciudadanos habitan en las zonas más modernas y más democráticas y otros en zonas más atrasadas y menos democráticas. Asimismo, las desigualdades sociales generan brechas ciudadanas por género, cultura, región, entre otros. Las brechas mencionadas son mayores en los departamentos menos modernos y menos democráticos. Por ejemplo, si se analiza la desigualdad social — de estatus y de clase - se encuentra que la ciudadanía reduce parcialmente la desigualdad de clase (desigualdad en el ingreso), pero mantiene la desigualdad en la propiedad de los activos (López 1997: 451).

La estructura de la desigualdad por un lado, y los movimientos sociales y políticos por otro, determinan un acceso diferenciado a los diversos tipos de derechos y a las prácticas ciudadanas. Donde no han existido movimientos sociales ni políticos, la estructura de la desigualdad y la voluntad de las élites detentadores del poder han cumplido un papel decisivo en el acceso o no a los derechos y prácticas ciudadanas. Tal es el caso de los derechos civiles y políticos en el Perú. Ante la ausencia de movimientos liberales y sufragistas, la concesión de los derechos civiles y políticos estuvo librada entre la pugna de las elites y las clases medias que asumían la canalización de las demandas de las clases populares. Es decir, dos elementos configuran las características de la ciudadanía en el Perú, por un lado la estructura de la desigualdad y por el otro, los movimientos sociales y políticos. El primer elemento es independiente a la propia ciudadanía, pero la segunda no. Sin embargo esta última se muestra a lo largo de la historia, débil e intermitente (Casalino 2010: 26). 
En refuerzo a lo señalado, López sostiene que es en el ámbito de las luchas de los sectores sociales donde se logra obtener los mayores avances en términos de derechos sociales (López 1997: 456). Por lo tanto, podemos observar que en el Perú, la desigualdad social, es un aspecto que incide directamente en la calidad de la ciudadanía y que las acciones sociales se han mostrado débiles y dispersas.

Si pasamos a la relación entre ciudadanía y pobreza, observamos que esta es muy clara en nuestro país. Para exponer sus características, es pertinente señalar en primer lugar, que la pobreza, tal como señala Sinesio López, es un fenómeno económico y social, por lo tanto no es un problema individual. López sostiene que entre la pobreza y la ciudadanía hay una relación inversa. Es decir, donde hay mayor pobreza se encuentra menos ciudadanía (López 1997: 456). Esta misma relación inversa se expresa incluso cuando analizamos la ciudadanía civil, la política y la social (López 1997: 462).

Una mirada más profunda respecto a esta relación entre pobreza y ciudadanía se puede realizar cuando se analiza la consistencia de la ciudadanía, en el sentido que el acceso a los recursos y las condiciones para ese acceso también son diferenciados:

Si recordamos la definición de ciudadanía como la totalidad de los miembros de un ente político común y su estatus como miembros de pleno derecho, y que ello se lleva a cabo a través de la nacionalidad. Entonces, es necesario reflexionar sobre lo que sucede en nuestro país, ya que la desigualdad y la pobreza impiden que un sector importante de la población pueda ser un ciudadano pleno, con acceso a los recursos y que pueda ejercer sus derechos. Fue con esa mirada que decidimos preguntar a jóvenes estudiantes de tres universidades de Lima, sobre sus percepciones sobre la ciudadanía. Los resultados los mostraremos en el siguiente acápite.

\section{Las percepciones de la ciudadanía en los jóvenes de tres universidades de Lima ${ }^{4}$}

El grupo Historia y Ciudadanía Activa llevó a cabo una encuesta para conocer la percepción de algunos estudiantes universitarios limeños acerca de temas de

4 Este acápite estuvo liderado por David Atarama y el equipo que participó aplicando las encuestas estuvo conformado por estudiantes de las tres universidades mencionadas, así como por integrantes del grupo de investigación Historia y Ciudadanía Activa (Madeleine Torres, Juan Marcos Martínez, Miguel Ccasani, Joel Segura, Joel Castro, entre otros) ciudadanía. Nuestro objetivo era conocer cuáles son las percepciones de las y los ciudadanos jóvenes respecto a su condición ciudadana y su compromiso con la construcción de la comunidad política. La encuesta se desarrolló en las siguientes tres casas de estudio: Universidad Nacional Mayor de San Marcos, Universidad Nacional Federico Villarreal y Universidad Antonio Ruiz de Montoya. A continuación presentamos los resultados obtenidos de la aplicación de estas encuestas.

La primera interrogante se planteó en torno el conocimiento básico de qué es lo que nos hace ciudadanos. Estos fueron los resultados:

Ante la pregunta, "Según la ley ¿qué se necesita para ser ciudadano? Se obtuvieron los siguientes resultados:

\begin{tabular}{lr}
\hline Opciones de respuesta & Resultados \\
\hline Ser mayor de 18 años & 809 \\
\hline Terminar los estudios básicos & 30 \\
\hline Votar por primera vez & 23 \\
\hline Nacer & 129 \\
\hline No sabe & 21 \\
\hline
\end{tabular}

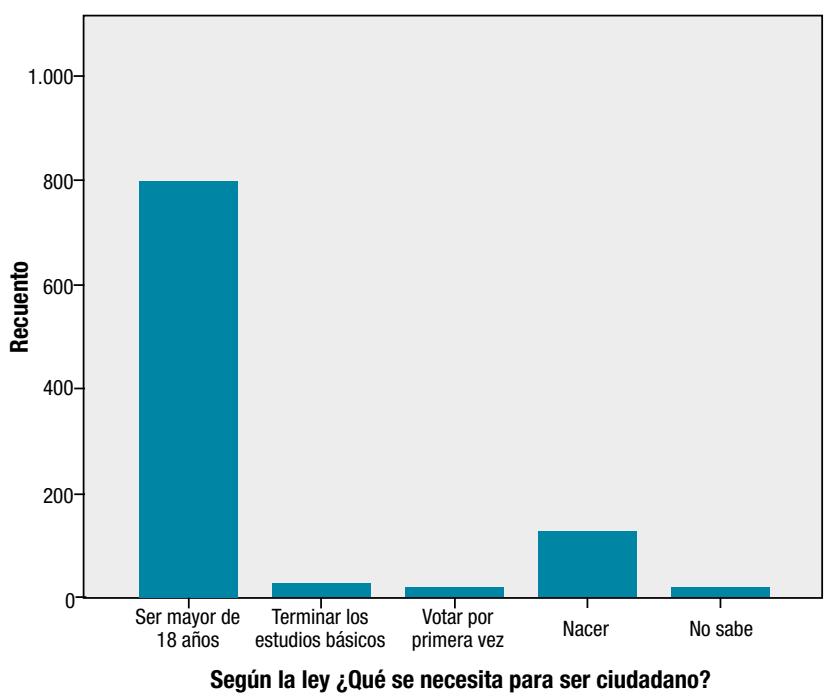

Como se puede observar no hubo problemas en cuanto al conocimiento de cómo una persona se convierte en ciudadana según el marco legal. Puesto que más del $80 \%$ supo responder correctamente. Ello significa que los jóvenes universitarios conocen lo dispuesto por la ley respecto a cómo se accede a ser ciudadano. En los casos en que se optó por la respuesta "nacer" ello se debería a que se tiene la información que al nacer se es sujeto de derecho.

Después de formular la interrogante sobre la información que el joven tiene sobre la norma respecto a ser ciudadano, nos interesó conocer las percepciones de los 
jóvenes respecto a la ciudadanía y la representación. De manera particular las interacciones entre el gobierno, los partidos y los procesos electorales. Así la pregunta y los resultados obtenidos fueron los siguientes:

Ante la pregunta “¿En qué medida consideras que la interacción entre el gobierno, los partidos y las elecciones te permiten participar y ejercer la ciudadanía? Se obtuvo los siguientes resultados:

\begin{tabular}{|l|r|}
\hline Opciones de respuesta & Resultados \\
\hline Muy poco & 103 \\
\hline Poco & 277 \\
\hline Regular & 505 \\
\hline Mucho & 126 \\
\hline Demasiado & 34 \\
\hline Total & 1045 \\
\hline
\end{tabular}

A través de la formulación de esta pregunta se quiso saber la percepción de los y las jóvenes sobre su participación política y el ejercicio de su ciudadanía en los procesos de selección de las autoridades y representantes. Aspecto que se puede considerar como lo básico para el ejercicio de los derechos políticos, ya que nuestras normas establecen determinadas garantías para que la ciudadanía política se ejerza no solo en periodo electoral. Los resultados nos señalan que las y los jóvenes universitarios consideran regular e insuficiente (poco y muy poco) el ejercicio de la ciudadanía cuando deben acercarse a las urnas para ejercer su voto y así elegir a las autoridades y a sus representantes.

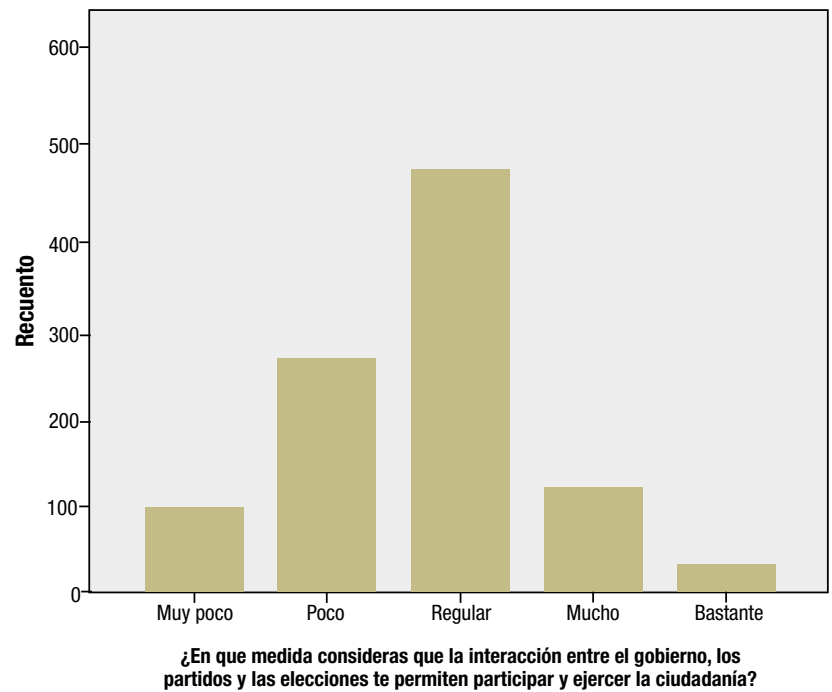

Luego nos interesó indagar sobre las percepciones y opiniones respecto al voto, saber qué pasaba si este era facultativo u obligatorio. En ese sentido, la pregunta formulada fue la siguiente: "Si las elecciones no fueran obligatorias ¿ejercerías tu derecho a voto?, obteniendo los siguientes resultados:

\begin{tabular}{lr}
\hline Opciones & Resultados \\
\hline Sí & 783 \\
\hline No & 200 \\
\hline No sabe & 65 \\
\hline Total & 1048 \\
\hline
\end{tabular}

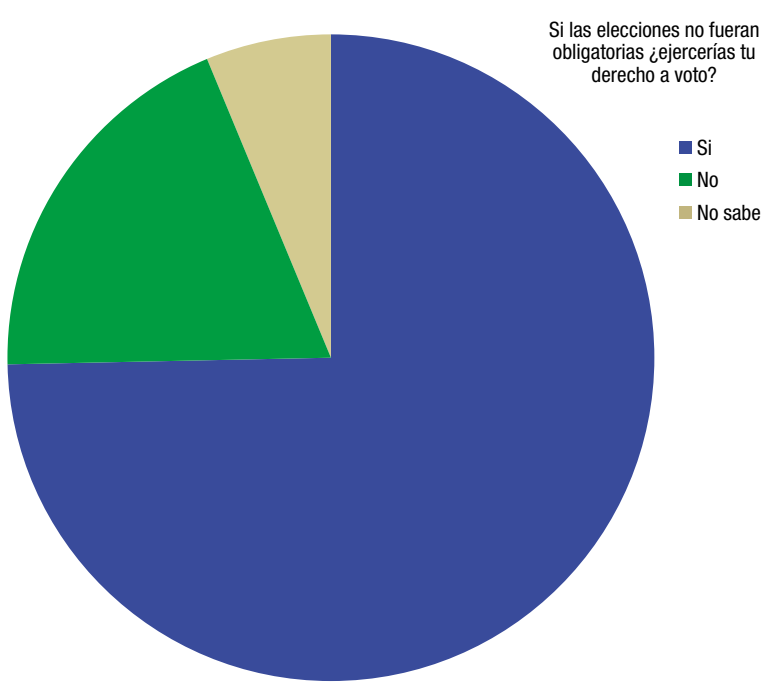

Como se puede observar los resultados son alentadores, puesto que el $75 \%$ de los encuestados votaría en el supuesto que no sean obligatorias las elecciones. Aunque esto debe analizarse bien y tener en consideración que las encuestas fueron desarrolladas por estudiantes universitarios, que le daría cierta característica a este resultado. Ello ratifica lo propuesto por Sinesio López que cuanto más acceso a la educación se tiene, se puede obtener más conciencia ciudadana y mayor predisposición para participar en los asuntos públicos. De ahí que la educación es fundamental para la formación y el fortalecimiento de la ciudadanía.

La siguiente pregunta buscó conocer el grado de representación que tiene el Estado según las percepciones de los jóvenes de las tres universidades de Lima encuestados.

En una escala de 1 al 5 ¿de qué manera te sientes representado por las instituciones del Estado?

\begin{tabular}{ccccc}
\hline Muy poco & Poco & Regular & Mucho & Bastante \\
\hline Recuento & Recuento & Recuento & Recuento & Recuento \\
\hline 240 & 382 & 332 & 49 & 12 \\
\hline
\end{tabular}

Como se puede observar, es muy grande la falta de representación que se tiene del Estado, esto podría ser 
un indicador del porcentaje de personas que practican su ciudadanía. Si bien la opción por elegir a las autoridades y a los representantes es alta. Es en el ejercicio del poder y de la representación que es necesario hacer los ajustes.

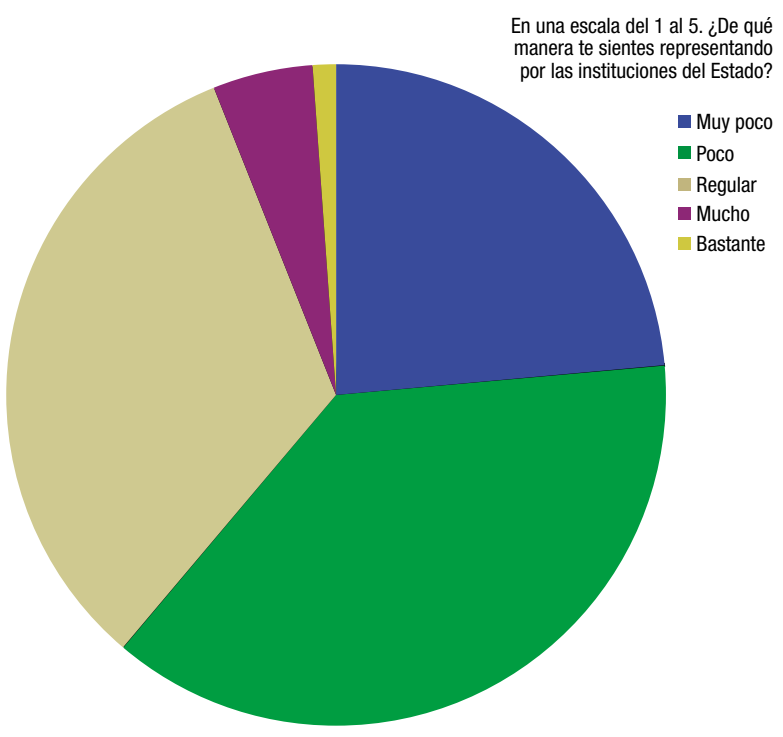

Ello significa que los electores jóvenes no dan un "cheque en blanco" cuando eligen, sino que conforme se va desarrollando la representación, estos jóvenes universitarios en su rol de ciudadanos-electores se van decepcionando de sus autoridades y representantes. Ello alimentaría el distanciamiento que se produce entre la sociedad y el Estado. Se va generando, a su vez, la sensación que los políticos han ofrecido a los electores un sinnúmero de propuestas solo con el fin de obtener los votos, pero no se sienten comprometidos una vez en el ejercicio de su función. Falta por lo tanto utilizar y activar los mecanismos de control y participación ciudadanos.

Las siguientes preguntas buscan conocer qué tan relevante es la política para los estudiantes universitarios. Así, una de las preguntas formuladas fue "¿Qué tan interesado estás en la política del país?”. Las respuestas obtenidas fueron las siguientes:

\begin{tabular}{|l|r|}
\hline Opciones de respuesta & Resultados \\
\hline Muy poco & 103 \\
\hline Poco & 133 \\
\hline Regular & 326 \\
\hline Mucho & 272 \\
\hline Bastante & 184 \\
\hline Total & 1018 \\
\hline
\end{tabular}

Se puede apreciar que los resultados fueron alentadores, puesto que el $75 \%$ de los estudiantes tenía una preocupación mediana hasta muy alta en lo que concierne a política. El grueso de las preferencias se manifiesta en regular, mucho y bastante. Son jóvenes universitarios que consideran que la ciudadanía es un ejercicio político.

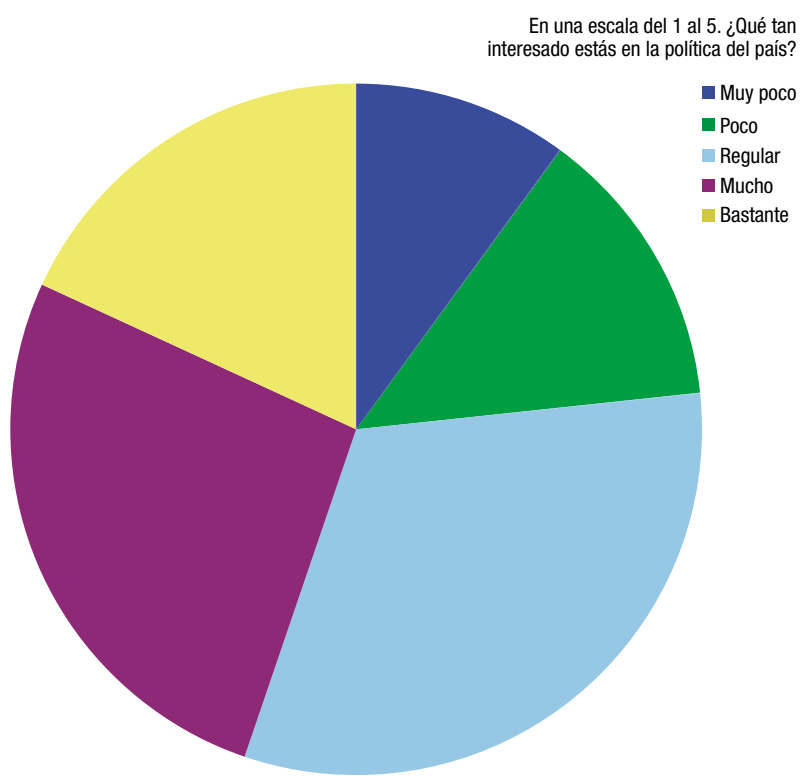

Decimos que estos resultados son alentadores, porque estos jóvenes han trascendido el prejuicio que la política es mala, tal como se expresaba en el discurso de la década de 1990, lo que dio lugar a una opción autoritaria y corrupta en el poder. También se puede observar que algunos están poco interesados y otros muy poco, ellos son lo que no han podido superar el discurso de la "antipolítica" que fue muy agresivo y que redujo a los ciudadanos a meros consumidores.

$\mathrm{Y}$ en la siguiente pregunta nos interesaba indagar sobre las percepciones que tienen los jóvenes sobre los elementos o condiciones que se requieren para participar en la política. Es decir, conocer si esas percepciones incidían si se trataba de un asunto predominantemente de recursos económicos, o sobre facultades personales que debían tener los políticos, o un asunto educativo, o una propuesta programática, entre otros.

Así, la pregunta fue formulada de la siguiente manera: “¿Qué recurso consideras que es el más importante para poder participar en política?”. Los resultados fueron los siguientes: 


\begin{tabular}{|l|r|}
\hline Opciones de respuesta & Resultados \\
\hline Dinero & 159 \\
\hline Influencias & 171 \\
\hline Grados académicos & 242 \\
\hline Carisma & 55 \\
\hline Propuestas & 279 \\
\hline Otras & 81 \\
\hline Ninguna & 22 \\
\hline
\end{tabular}

\begin{tabular}{|l|r|}
\hline Opciones de respuesta & Resultados \\
\hline Deportiva & 70 \\
\hline Social & 216 \\
\hline Cultural & 124 \\
\hline Religiosa & 77 \\
\hline Política & 46 \\
\hline Ninguna & 467 \\
\hline Más de una & 13 \\
\hline Total & 1013 \\
\hline
\end{tabular}

Como se puede apreciar, muchos quieren recibir las propuestas de los políticos y asegurar sus buenas prácticas en base de sus grados académicos. Pero se debe tener en cuenta que varios también tienen en consideración el dinero y las influencias para participar en política, lo cual es preocupante. En primer lugar, porque en este país no hay financiamiento a los partidos políticos, y tampoco hay regulación para garantizar que en la competencia electoral todos compitan en igualdad de condiciones. En segundo lugar, es preocupante, porque esa falta de financiamiento público, hace que algunos opten por buscar financiamiento que está condicionado a obtener beneficios una vez que se llega al poder, comprometiendo de esa manera su propuesta de gobierno a intereses particulares, o se llevan a cabo compromisos ilícitos.

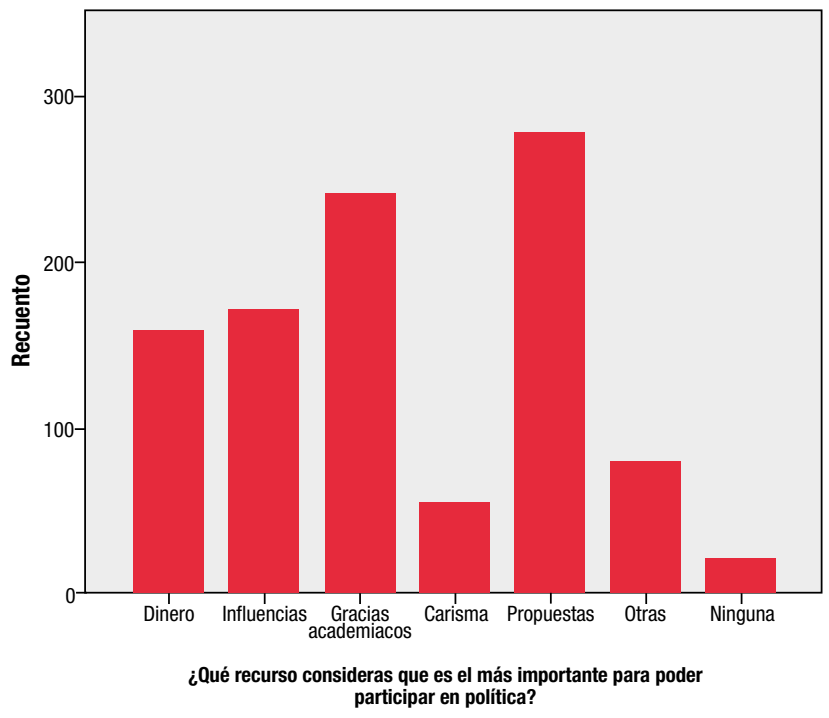

En el siguiente gráfico se ilustran los resultados sobre las organizaciones a las que suelen pertenecer los jóvenes universitarios. En ese sentido la pregunta formulada fue "¿A qué tipo de organización perteneces?. Las opciones de respuesta y sus resultados fueron:

Lo primero que se puede apreciar es que casi la mitad no están organizados en alguna agrupación. Si bien los jóvenes suelen ir juntos o preferir la compañía de otros jóvenes, estas asociaciones no son institucionalizadas, sino que están en el ámbito de la amistad. Sin embargo, los que optan por ingresar a algún tipo de organización, prefieren las organizaciones sociales y a una distancia mayor están las organizaciones culturales y religiosas.

En la siguiente gráfica se puede visualizar estos resultados:

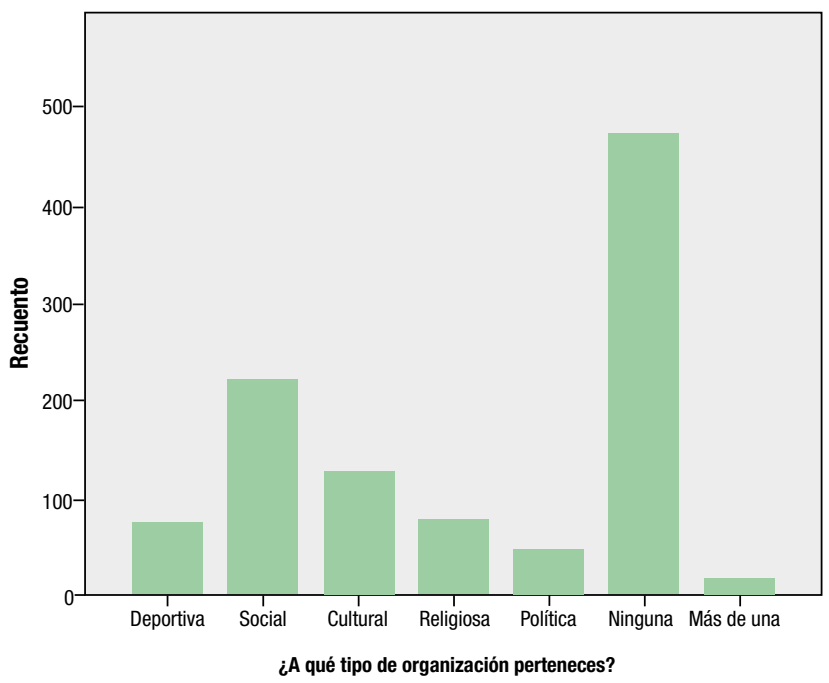

La siguiente pregunta buscó indagar en las percepciones de los jóvenes, de qué manera ellos sentían que contribuían a mejorar su comunidad, a través de qué acciones puntuales. En ese sentido, la pregunta que se les formuló fue: "¿Qué acciones realizas para la mejora de tu comunidad?. El resultado de dicha pregunta fue el siguiente: 


\begin{tabular}{|l|r|}
\hline Opciones de respuesta & Resultados \\
\hline Utilizo mecanismos de representación & 125 \\
\hline Estudiando de manera consciente y responsable & 473 \\
\hline Siendo candidato en procesos electorales & 16 \\
\hline Ejerciendo el derecho al voto & 174 \\
\hline Otras & 110 \\
\hline Ninguna & 66 \\
\hline Realizo más de una acción de las señaladas & 52 \\
\hline
\end{tabular}

¿Qué acciones realizas para mejora de tu comunidad?

- Mecanismos de representación

- Estudiando de manera consciente y responsable

Siendo candidato en procesos electorales

- Ejerciendo el derecho a voto

Otras

- Ninguna

- Más de una

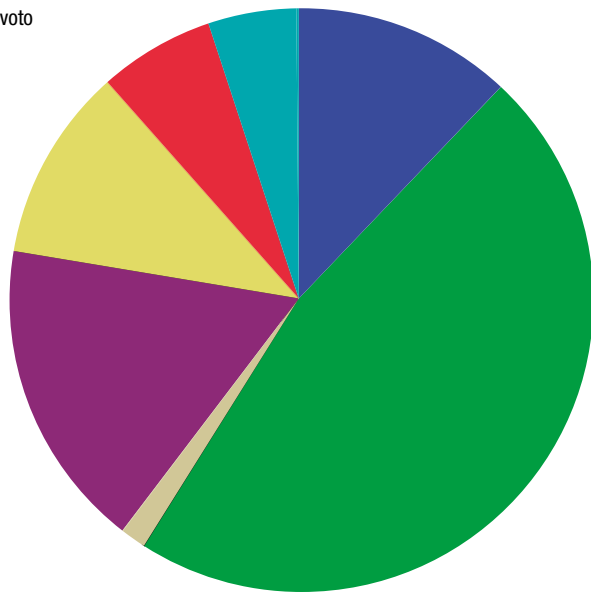

Los resultados revelan que los jóvenes consideran que cumpliendo a cabalidad su rol básico de ser estudiantes universitarios están contribuyendo con su comunidad. Es decir, creen que los estudios influenciarán en la mejora de la comunidad, otra vez se tiene que tener en consideración que los encuestados son estudiantes universitarios que se están formando para ser profesionales, momento en el cual piensan que tomarán decisiones que involucren o que tengan efectos en los demás.

Finalmente, se les preguntó sobre algunos problemas estructurales de nuestro país para averiguar si ellos consideraban si el conocimiento de esos problemas podría intervenir en la formación de su ciudadanía. En ese sentido, la pregunta formulada fue: "En tu opinión, ¡el conocimiento sobre la violencia política de la década de 1980, o los temas de corrupción de la década de 1990, tienen relación con la formación de la ciudadanía?". Lo que dio los siguientes resultados:

\begin{tabular}{|l|r|}
\hline Opciones de respuesta & Resultados \\
\hline Sí & 891 \\
\hline No & 96 \\
\hline No sabe. No opina & 61 \\
\hline
\end{tabular}

La respuesta fue contundente, una amplia mayoría consideraba que conocer los problemas estructurales como el conflicto armado interno o la corrupción, que aparentemente fueron flagelos de las décadas de 1980 y 1990, están relacionados con la formación de la ciudadanía.

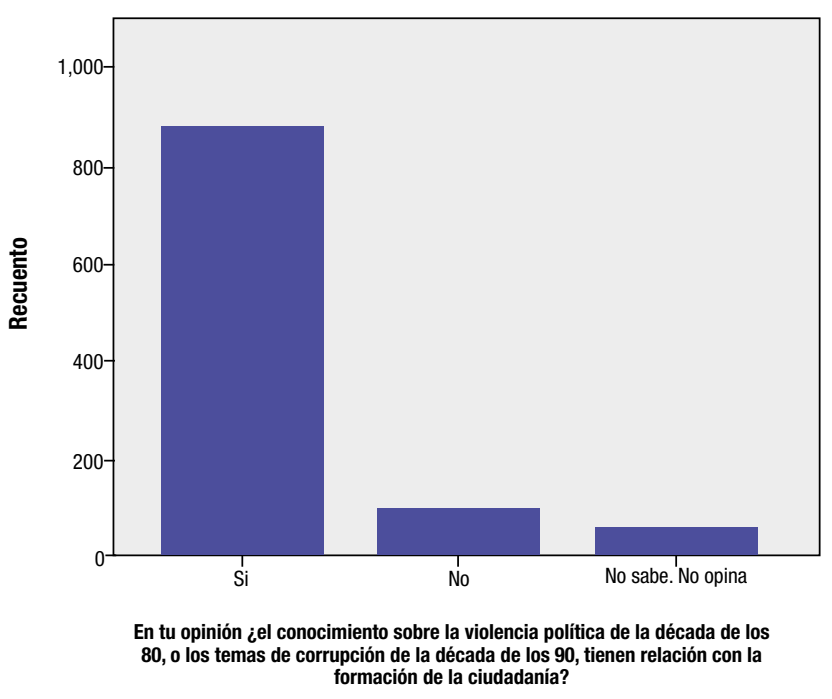

Tomando en consideración los objetivos de nuestra investigación, en el cuestionario, buscamos profundizar las respuestas anteriores, para tener una mejor orientación respecto a nuestro quehacer. Por esa razón, para los que contestaron "si", les hicimos una repregunta: "Si tu respuesta es sí, ¿en qué medida crees que el conocimiento de estos temas permiten fortalecer tu ciudadanía?. Las opciones de respuesta fueron una escala del 1 al 5 siendo el 1 el de menos grado y 5 el de mayor grado. Así, las respuestas fueron las siguientes:

\begin{tabular}{|l|r|}
\hline Opciones de respuesta & Resultados \\
\hline $1=$ muy poco & 25 \\
\hline $2=$ poco & 69 \\
\hline $3=$ regular & 217 \\
\hline $4=$ mucho & 319 \\
\hline $5=$ bastante & 272 \\
\hline
\end{tabular}

Dado que los problemas del conflicto armado interno y la corrupción son temas históricos, consideramos que los estudiantes señalaron que estos temas históricos que son problemas estructurales, son importantes de ser conocidos para fortalecer su ciudadanía. Lo que se aprecia mejor en el siguiente gráfico: 


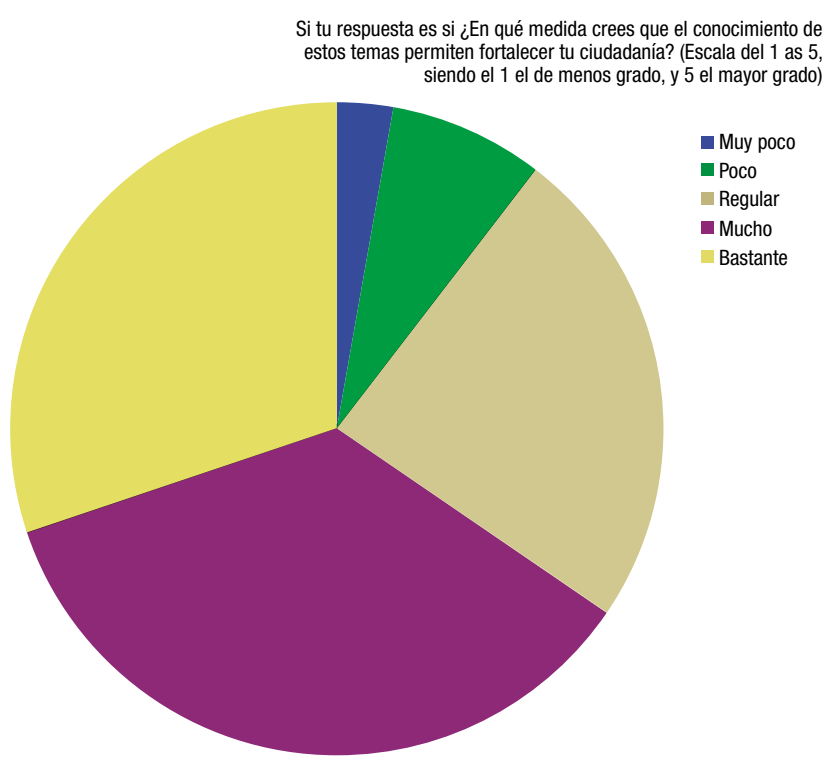

Con lo cual consideramos que la ciudadanía, en los jóvenes de las tres universidades participantes, era vista con interés. Además, que el conocimiento de problemas históricos podía ayudarlos a fortalecer su ciudadanía.

\section{Conclusiones}

En este breve ejercicio hemos visto que países como los nuestros, donde predominan las instituciones políticas extractivas, es urgente desarrollar ciudadanía, para que sean los propios actores sociales y políticos los que busquen realizar los cambios que se requieren para poder dar oportunidades a gran parte de la población. Asimismo, hemos abordado la importancia de la educación, especialmente desde la perspectiva republicana, al entender la educación como motor de progreso y clave para el fortalecimiento de una ciudadanía virtuosa. Además, la enseñanza de la Historia contribuye a formar la identidad personal e individual, el propio yo que tiene directa relación con los valores intrínsecos de la persona. En ese sentido, podemos apreciar que la Historia también puede ayudar a fortalecer la ciudadanía, especialmente en jóvenes universitarios, quienes se preocupan y están interesados en conocer más sobre los problemas actuales del país que surgieron desde los inicios de la república, por ejemplo la corrupción. También están interesados en conocer más sobre la defensa de los derechos humanos y el conflicto armado interno. Finalmente, si bien no buscan organizarse, sí están interesados en movilizarse por aquello que rechazan.

\section{Referencias bibliográficas}

Acemoglu, Daron \& James Robinson (2012). Por qué fracasan los paises. Los origenes del poder, la prosperidad y la pobreza. Espańa: Deusto.

Audigier, F. (1999). L'éducation à la citoyenneté. Paris. INRP

Buriano, Ana (2005). La historia escolar y la historia académica. Algunas reflexiones en la era del "fin de la historia”. En: Correo del maestro, N. o 104, Año 2005. http:// www.correodelmaestro.com/pruebas/anteriores/2005/ enero/incert104.htm (última consulta 26/12/13).

Calaf Masachs, Rose (1994). Didáctica de las ciencias sociales: didáctica de la historia. España: Oikos-tau.

Cardona Zuleta, Luz Margarita (2011)."La noción republicana de virtud: de la virtud moral a la virtud cívica”. En: Forum N. ${ }^{\circ}$ 2, julio-diciembre 2011. Revista del Departamento de Ciencia Política Universidad Nacional, Sede Medellín: Colombia.

Casalino, Carlota (2010). Ciudadanía y Sociedad Civil. Elementos para comprender el caso peruano. Cuadernos de divulgación de educación electoral y democracia $\mathrm{N} .^{\circ} 3$. Lima: ONPE.

Cueva, Nicolás (1991). La enseñanza de la historia: teorias y técnicas. Lima: Centro de Investigación y Promoción del Campesinado CIPCA

Chávez García, Teresa (2006). La enseñanza de la historia del Perú en la educación secundaria durante la segunda mitad del siglo XX. Lima: PUCP.

Chumpitaz Campos, Lucrecia (2010). Didáctica para procesos educativos no escolares. Lima: PUCP-Facultad de Educación.

Del Águila, Alicia (2013). La ciudadanía corporativa. Politica, constituciones y sufragio en el Perú (1821-1896). IEP: Lima.

Del Valle Ballón, Roxana (2012). Fundamentos psicopedagógicos del aprendizaje adulto. Lima: PUCP-Facultad de Educación.

Eguren, Mariana, De Belaunde, Carolina y Natalia GonzÁLEZ (2003). "Repensando el texto escolar desde su uso: un diagnóstico para la escuela urbana”. [Versión Adobe Digital Editions]. http://old.cies.org.pe/files/documents/ investigaciones/educacion/repesando-el-texto-escolardesde-su-uso-un-diagnostico-para-la-escuela-urbana.pdf

Escobar, Patricia (1991). Fundamentos teóricos para una enseñanza creativa de la historia. Lima: PUCP-Tesis.

Fernández-Llebrez González, Fernando (2012). "Humanismo, participación y ciudadanía cívica. Elementos para una democracia de calidad". En: Revista Española de Ciencia Politica N. ${ }^{\circ}$ 30, noviembre 2012, pp. 31-53. 
Fernández Maldonado, Enrique (2015).La rebelión de los pulpines. Jóvenes, trabajo y política. Lima: Otra Mirada.

Ferro, Marc (1998). Cómo se cuenta la historia a los niños en el mundo entero. México: FCE.

Finocchietti, Susana (Comp.)(1999). Enseñanza de la historia y cultura de paz. Lima:

Fontana, Josep (1999). Enseñar historia con una guerra civil por medio. Daniel G. Linacero.- Mi primer libro de historia (1933). Instituto de España.- Manual de Historia de España (1939). Barcelona: Crítica.

González, María Paula (2011). "Saberes académicos y saberes escolares: para una revisión del concepto "transposición didáctica” desde la enseñanza de la historia. (última consulta: 26-12-13).

Gutiérrez, Carlos José (2000). "Ciudadanía” En: CAPEL. Diccionario electoral. IIDH/AECI: Costa Rica, tomo I, pp. 170-179.

IguiÑIz, Manuel \& Arturo Miranda (2011). La educación de los ciudadanos. Política educativa en Lima metropolitana. Lima: Tarea.

Lima, Laura; Felipe Bonilla \& Verónica Arista (2010). La enseñanza de la historia en la escuela mexicana. México: Proyecto Clio 36. (última consulta: 26-12-13).

López, Sinesio (1997). Ciudadanos reales e imaginarios. Concepciones, desarrollo y mapas de ciudadanía en el Perú. Instituto Diálogo y Propuestas: Lima.

Marshall, T. H. (1965). "Citizenship and Social Class". En: T. H. Marshhall (comp.) Class, Citizenship and Social Development. Doubleday: New York.

Meza BazÁn, Mario (2013). Justicia y poder en tiempos de violencia. Orden, seguridad y autoridad en el Perú (19702000). Lima: PUCP.

Ortega Ruiz, Rosario (2005). Psicología de la enseñanza y desarrollo de personas y comunidades. México: FCE.
Pease García, Henry \& Gonzalo Romero Sommer (2013). La politica en el Perú del siglo XX. Lima: PUCP.

Peña, Javier (2000). "El retorno de la virtud cívica” En: José Rubio Carracedo et al. Educar para la ciudadanía. Perspectivas ético-políticas. Suplemento N. ${ }^{\circ}$ 8. Contrastes Revista Internacional de Filosofía. Universidad de Málaga: Málaga (pp. 81-105).

Peralta, Víctor (2010). La independencia y la cultura política peruana (1808-1821). Lima: IEP-Fundación Bustamante de la Fuente.

Quiroz, Alfonso (2013). Historia de la corrupción en el Perú. Lima: IEP-IDL.

Rieger, Günter (2006). "Ciudadanía” en: Dieter Nohlen. Diccionario de Ciencia Política. Teorías, métodos, conceptos. Editorial Porrúa-El Colegio de Veracruz: México. Tomo I, pp. 203-204.

Rojas Galarza, Carlos (1989). La enseñanza de la historia y otras reflexiones acerca de educación y politica. Lima: El Alba

Ruiz Ruiz, Ramón (2009). "La concepción republicana de la democracia y de la virtud pública”. En: Presente, pasado y futuro de la democracia, 2009, pp. 75-82.

Salmerón, Ana María (2006). "Entre liberalismo y republicanismo. El lugar de la virtud cívica en el ordenamiento social y educativo”. En: Trayectorias, vol. VIII, núm. 22, septiembre-diciembre, 2006, pp. 56-65. Universidad Autónoma de Nuevo León: México.

Savater, Fernando (2004). El valor de educar. Barcelona: Ariel.

Valladares Quijano, Manuel (2013). El Paro Nacional del 19 de julio. Movimientos sociales en la época del "Gobierno Revolucionario de las Fuerzas Armadas". Lima: UNMSM-Pakarina.

VAlle, Augusta (2013). "El conocimiento histórico y la escuela”. En: PUCP. Mural de Letras, Año 8, N. 13. 